\title{
Acquired Complete Heart Block with Long QT Interval and Recurrent Polymorphic Ventricular Tachycardia: A Case Report
}

\author{
Hosam Zaky, Jassem Al Hashmi \\ Dubai Hospital, Dubai, The United Arab Emirates \\ Email: hzaky64@gmail.com, jmalhashmi2@gmail.com
}

Received 21 April 2016; accepted 13 June 2016; published 16 June 2016

Copyright (C) 2016 by authors and Scientific Research Publishing Inc.

This work is licensed under the Creative Commons Attribution International License (CC BY). http://creativecommons.org/licenses/by/4.0/

(c) () Open Access

\begin{abstract}
We are reporting a case of acquired complete heart block and long QT interval (a dispersion of repolarization that leads to polymorphic ventricular tachycardia) that has presented with loss of conscious and proved to be due to torsade de pointes. The patient responded well to cardiac pacing and beta blocker therapy. The association of complete acquired heart block and long QT interval is quite rare.
\end{abstract}

\section{Keywords}

\section{Heart Block Long QT}

\section{Introduction}

Long QT syndrome (LQTS) is an uncommon cardiac disorder of repolarization leading to prolonged QT interval and T-wave abnormalities on the ECG, thus predisposing to Torsades de Pointes (TdP) and sudden cardiac death [1]. A study by Schwartz et al. [2] reported the prevalence of LQTS to be 1 in 2000 people. Pseudo 2:1 AV block when sinus intervals are shorter than the ventricular refractory period has been reported with long QT syndrome (LQTS) [2], but only few reports of acquired complete heart block, and few reports of congenital complete heart block with prolonged QT interval [3]-[6].

\section{Case Report}

We report a 29-year-old gentleman, with positive family history of sudden cardiac death, and no cardiac symptoms before, who presented to our center with syncope, his ECG showed complete heart block with an escape 


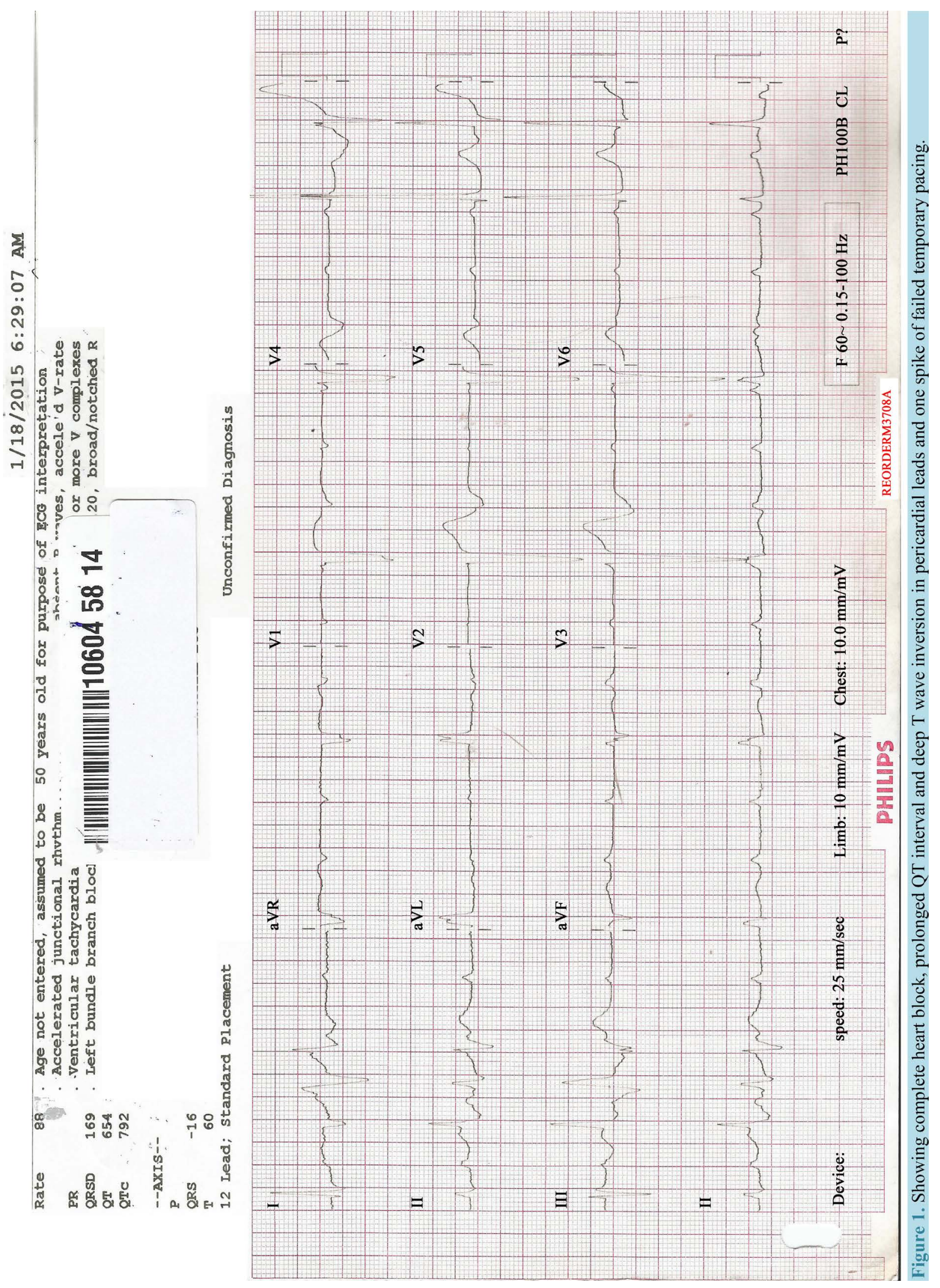



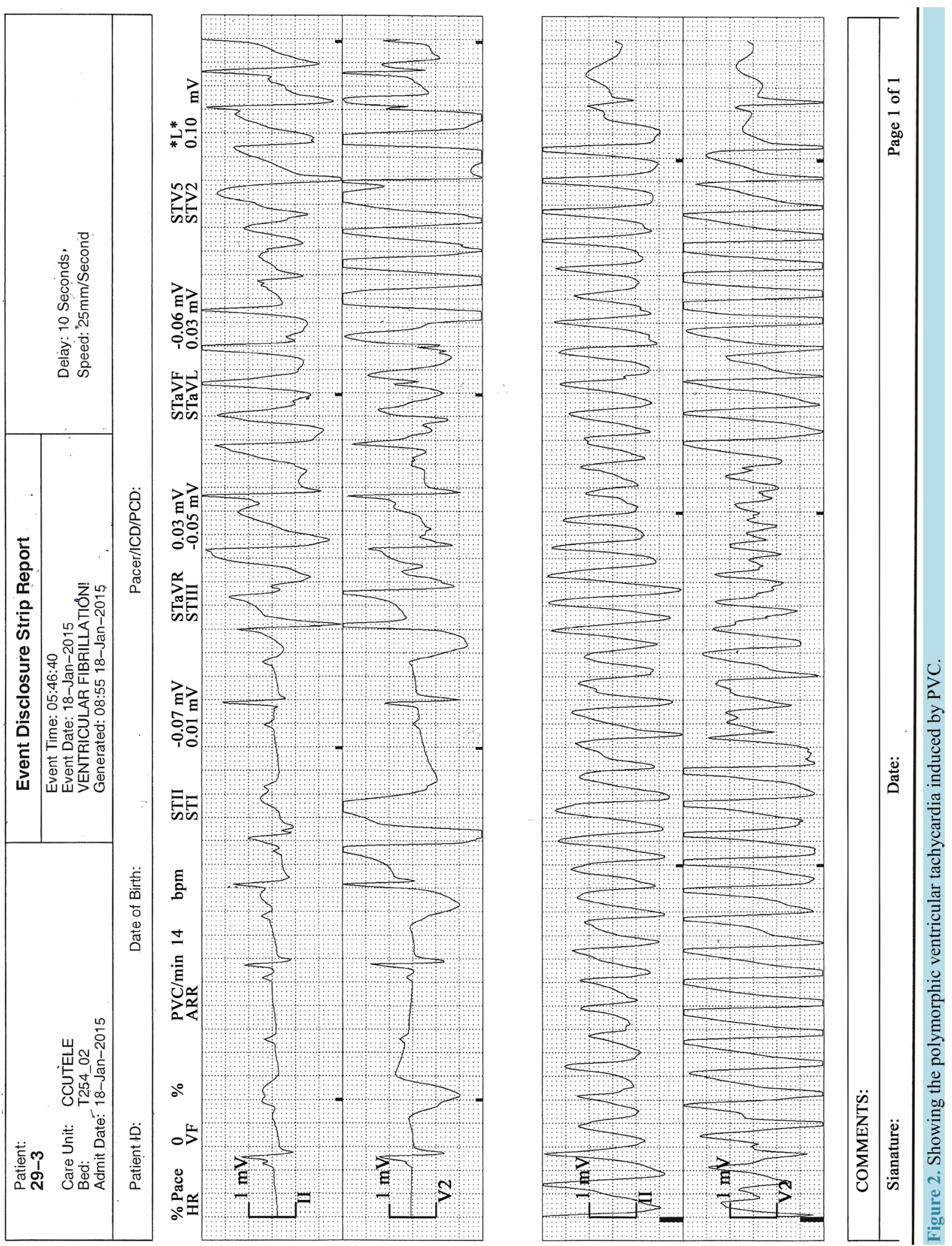

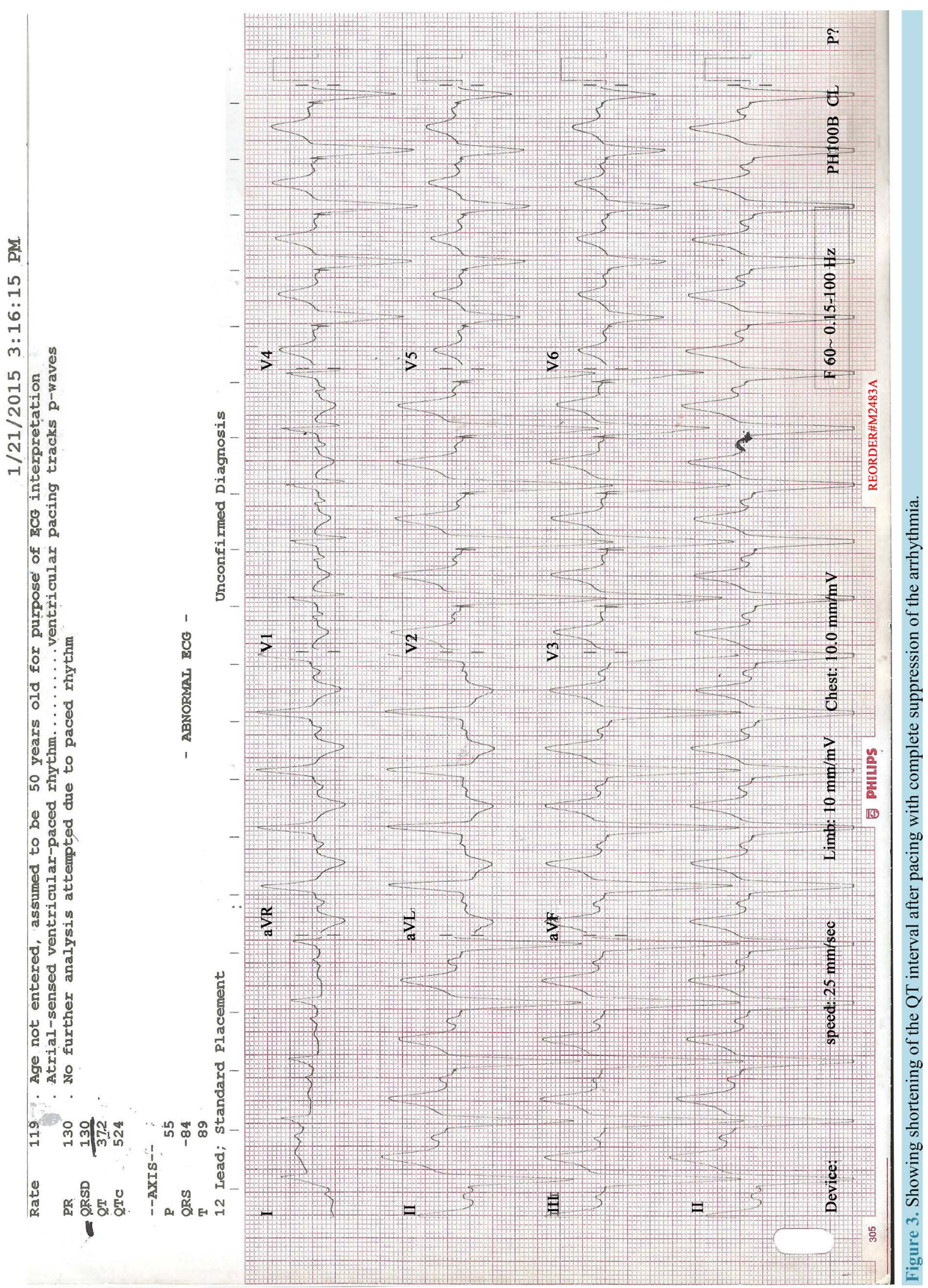
rhythm of wide QRS, at a rate of 35 beats per minutes, We had no previous records of the patient as he has recently arrived to the country but this was his first time to have such a complaint, inpatient work up did not reveal any reversible cause of complete heart block, his QT interval was prolonged (QT $680 \mathrm{~ms}$, QTc bazett method $534 \mathrm{~ms}$, QTc Hodges method $640 \mathrm{~ms}$ ) (we used the Hodges method because the patient was tachycardiac after DDD pacing as the bazett method would overestimate the corrected QT when the patient heart rate above 110 BPM [6]), with deep T wave inversion in the precordial leads (Figure 1). On telemetry he started to have recurring polymorphic ventricular tachycardia which was initiated by a premature ventricular beat (Figure 2) and led to loss of consciousness. A temporary pacemaker was inserted which suppressed the arrhythmia and led to shortened QT. His echocardiogram, serum electrolyte including potassium and coronary angiogram were normal. A permanent dual chamber pacemaker was implanted the following day with higher basal rate (Figure 3) and the patient was started on beta blocker. QT and QTe were significantly reduced after permanent pacing (QT 400 ms QTc bazett 573 ms (overestimated as heart rate is 119 BPM), QTc Hodges 503 ms) [7]. He remained arrhythmia free and discharged in good condition. At six weeks follow up the patient was seen doing well, without symptoms or recorded arrhythmia, and normal pacemaker function.

\section{Discussion}

The ventricular tachyarrhythmia that underlies the cardiac events of LQTS is Torsades-de-Pointes, a curious type of ventricular tachycardia that most of the time is self-limiting and produces transient syncope but that can also degenerate into ventricular Fibrillation and cause cardiac arrest or sudden death [1]. The association of acquired complete heart block and long QT interval in literature is quite rare, only few reports about congenital complete heart block and long QT interval [3] [4]. Indik [8] had also reported a similar case study of a 65-years old female who developed dizziness and found to have complete heart block with QT interval of $800 \mathrm{~ms}$ and markedly abnormal $\mathrm{T}$ wave. She was demonstrated to have episodes of TdP on Holter monitoring. After insertion of the pacemaker, the T-waves normalized and QT interval shortened and arrhythmia suppressed. It is generally accepted that in the long QT syndrome, the irritability of the ventricle increases due to the exaggerated dispersion of ventricular repolarization and unilateral changes in sympathetic tone of the heart (functional distribution of right and left stellate innervation to the ventricles). The appearance of polymorphous ventricular tachycardia mostly of the torsade de pointes type can frequently be detected in the syndrome of QT prolongation. Contrary to congenital complete heart block, the acquired complete heart block can have more frequent attacks of loss of conscious. In our work, we went in agreement with other authors [3]-[6] that pacing shortened the QT interval and prevented the provocation of the torsade de pointes. In searching for the etiology of our condition, a study by Kurita et al. [8] investigated fourteen patients with complete atrioventricular block. They demonstrated that there was significant prolongation of the QT and QTc intervals among patients with complete heart block who developed TdP as compared to those who did not develop TdP: $753 \pm 57.5 \mathrm{vs} 635 \pm 78.4 \mathrm{~ms}(\mathrm{p}<0.01)$ and $585 \pm 44.8$ vs $476 \pm 58.3 \mathrm{~ms}(\mathrm{p}<0.01)$ which might point to the mechanism of arrhythmia in our case.

\section{References}

[1] Schwartz, P.J., Crotti, L. and Insolia, R. (2012) Long QT Syndrome: From Genetics to Management. Circulation: Arrhythmia and Electrophysiology, 5, 868. http://dx.doi.org/10.1161/CIRCEP.111.962019

[2] Schwartz, P.J., Stramba-Badiale, M., Crotti, L. and Pedrazzini, M. (2009) Prevalence of the Congenital Long-QT Syndrome. Circulation, 120, 1761-1767.

[3] Gascho, J.A. and Schieken, R. (1979) Congenital Complete Heart Block and Long Q-T Syndrome Requiring Ventricular Pacing for Control. of Refractory Ventricular Tachycardia and Fibrillation. Journal of Electrocardiology, 3, 331335.

[4] Solti, F., Szatmary, L., Vecsey, T., Renyi-Vamos Jr., F. and Bodo, E. (1992) Congenital Complete Heart Block Associated with QT Prolongation. European Heart Journal, 13, 1080-1083.

[5] Rajani, A.R., Hussain, K., Radaideh, G.A. and Agarwal, S.K. (2012) Long QT Syndrome in a Patient with Complete Heart Block Causing Torsades de Pointes. Pakistan Journal of Medical Sciences, 28, 512-515.

[6] Indik, J.H. (2008) Not Just Slow: Double Trouble. American Journal of Medicine, 121, 19-20. http://dx.doi.org/10.1016/j.amjmed.2007.08.006

[7] Qiu, H., Bird, G.L., Qu, L., Vetter, V.L. and White, P.S. (2007) Evaluation of QT Interval Correction Methods in Normal Pediatric Resting ECGs. Computers in Cardiology, 34, 431-434. http://dx.doi.org/10.1109/cic.2007.4745514 
[8] Kurita, T., Ohe, T., Marui, N., Aihara, N., Takaki, H., Kamakura, S., et al. (1992) Bradycardia-Induced Abnormal QT Prolongation in Patients with Complete Atrioventricular Block with Torsades de Pointes. American Journal of Cardiology, 69, 628-633. http://dx.doi.org/10.1016/0002-9149(92)90154-Q 